\title{
Morphologie et transport solide de rivières à forte pente
}

\author{
M. Ramette Société Sofratome, Rueil-Malmaison .
}

Ces dernières années, des études sur modèle ont été entreprises par la Sogreah pour l'aménagement de rivières à forte pente dans l'Ile de la Réunion pour le compte de la DDE de ce Département. En particulier la "Rivière des Galets " a fait récemment l'objet d'une étude de divers types d'endiguement sur modèle à l'échelle du 1/125 (réf. 0). L'étalonnage du modèle qui a précédé les études d'endiguement, donne des relations débits liquides-débits solides qui, compte-tenu de l'échelle utilisée et du soin apporté à l'étalonnage, peuvent être considérées comme très fiables, au moins pour les fortes valeurs du débit.

Il a paru intéressant de confronter ces relations expérimentales avec celles qui se déduisent des études théoriques morphologiques entreprises par ailleurs (réf. 1): un ajustement de certains paramètres et la prise en compte d'un charriage sur fond plat permettent d'obtenir une comparaison satisfaisante entre expérience et théorie. Ces lois théoriques établies entre débits liquides et débits solides, associées aux études hydrologiques conduites par ailleurs, peuvent aider à définir l'ordre de grandeur des apports solides moyens annuels et de ce fait contribuer, par exemple, à définir une politique d'extraction des graviers. Elles sont par ailleurs utiles pour dégrossir les caractéristiques d'un éventuel endiguement respectant au mieux la morphologie de la rivière naturelle.

\section{Rappel des notations utilisées}

\subsection{Paramètres : \\ $S \quad$ pente de la ligne d'énergie \\ $Q_{L} \quad$ débit liquide $\left(\mathrm{m}^{3} / \mathrm{s}\right)$ \\ QS débit solide $\left(\mathrm{m}^{3} / \mathrm{s}\right)$ \\ $W \quad$ largeur du lit $(\mathrm{m})$}

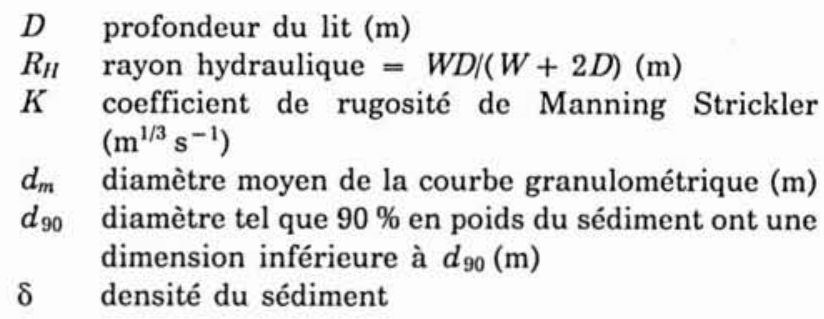

\section{Morphology and bed-load transport of steep sloped rivers}

These last few years, model studies were carried out the Sogreah for the development of steep sloped rivers on the island of Réunion. The calibration - of the model of the "Rivière des Galets", which precedes damming up research, provides the relationships between bed-loads and liquid flows which, having regard to the scale used and the care involved in the calibration, may be considered as quite valuable, at least for flowrate high values. These experimental relations are compared with those deduced from theoretical morphological studies undertaken elsewhere (ref. 1): the adjustment of certain parameters and the taking into account of a flat bed transport enable a comparison to be obtained between experiment and theory.

These theoretical laws established between liquid flows and solid flows, allied to hydrological studies conducted elsewhere, may assist in defining the order of magnitude of mean annual solid supplies. 


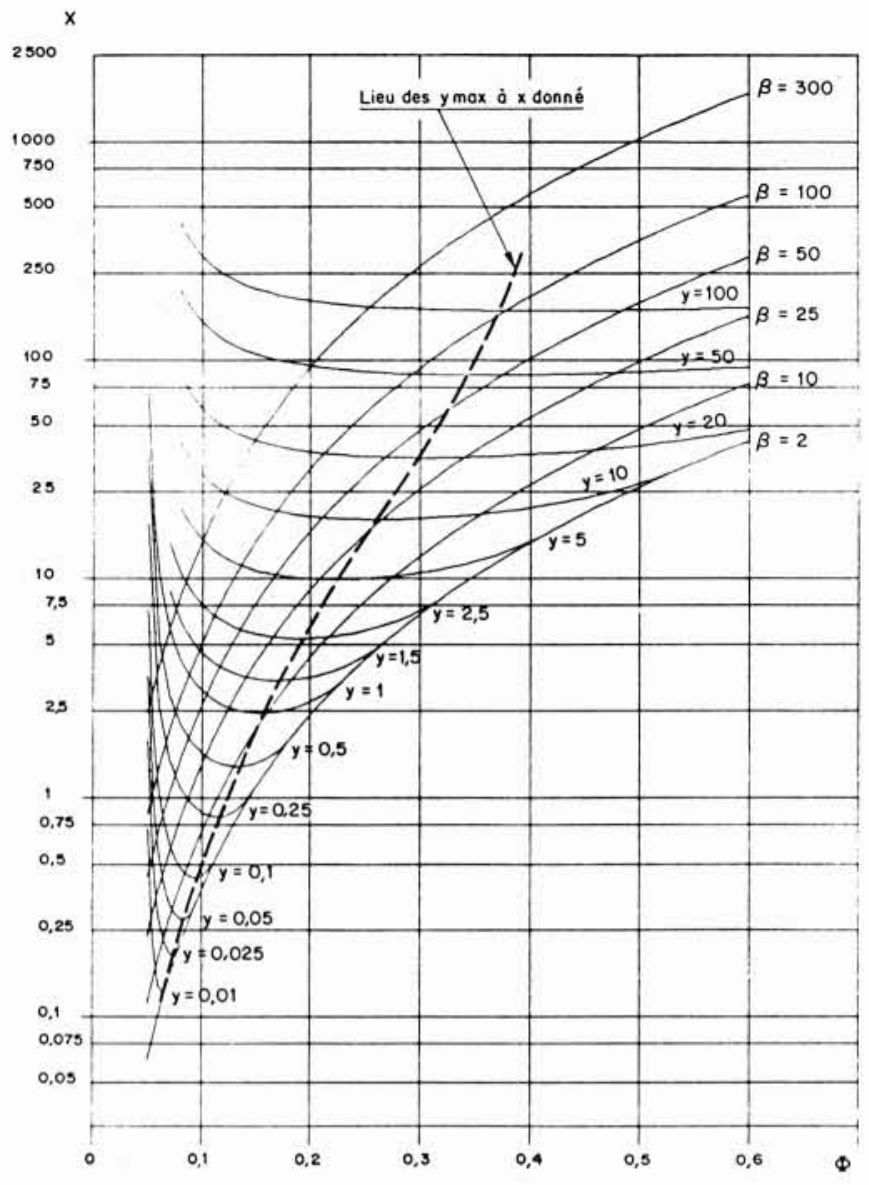

1. $\alpha=1$.

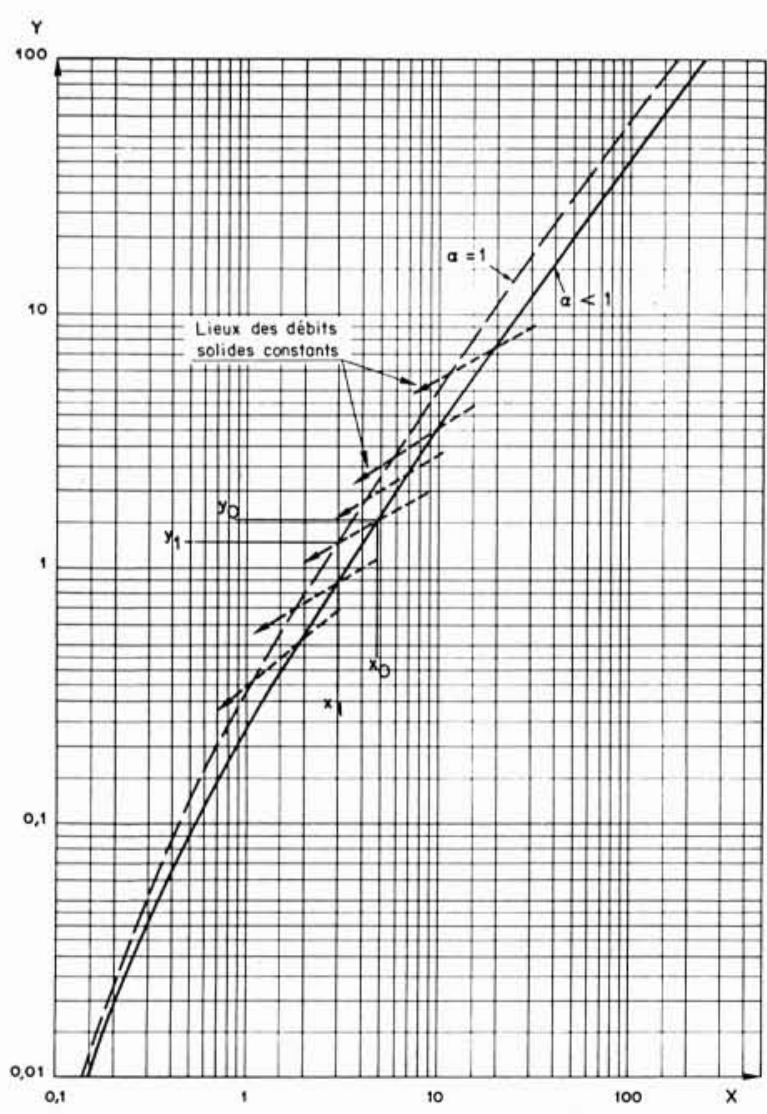

2. Débit solide maximum: $Y=f(x)$.

\subsection{Coefficients sans dimension :}

$\alpha=$ coefficient minorateur de $S$

$\beta=W / D$

$\phi=R_{H} S(\delta-1) d_{m}$

\section{Hypothèses prises en compte}

\subsection{Maximum de débit solide}

La condition de stabilité du lit conduit à maximiser le transport solide pour un débit liquide donné (Référence 1).

\section{Qs maximum}

\subsection{Transport solide}

Le transport solide s'effectue par charriage et sur fonds plats, quel que soit le débit.

L'expression du charriage est donnée par la relation de Meyer-Peter Müller, en l'absence de dunes (coefficient minorateur $\alpha$ proche de 1 ).

L'absence de dunes dans le type de rivières étudiées sera justifiée dans le cours du texte.

\section{3. Équations utilisées}

\subsection{Ecoulement liquide}

L'écoulement obéit à une loi du type Manning-Strickler

$$
\underline{Q_{L}}=K W D R_{H}^{2 / 3} S^{1 / 2}
$$

avec $K$ coefficient de rugosité donné par

$$
K=\left(21 / m_{m}^{1 / 6}\right)\left(d_{m} / D\right)^{1 / 24}
$$

Cette relation, classique, rend compte assez correctement des valeurs expérimentales tirées des essais sur le modèle de la Rivière des Galets.

L'introduction des coefficients sans dimension donnés ci-dessus conduit à l'expression suivante, en groupant les données dans le $1^{\text {er }}$ terme :

$Q_{L} S^{17 / 8} /\left(d_{m}^{5 / 2}(\delta-1)^{21 / 8}\right)=$

$$
X=21 \phi^{21 / 8}(\beta+2)^{47 / 24} /\left(\beta^{23 / 24}\right)
$$

\subsection{Débit solide}

La formule de Meyer-Peter Müller dans laquelle le débit solide est exprimé en $\mathrm{m}^{3}$ et en supposant une densité apparente de $1,8\left(1 \mathrm{~m}^{3}\right.$ pèse $\left.1,810^{4} \mathrm{~N}\right)$ s'écrit :

$$
\underline{Q_{S}}=13,7 \delta(\delta-1)^{3 / 2} W d_{m}^{3 / 2}[\alpha \phi-0,047]^{3 / 2}
$$

L'introduction des coefficients sans dimension donnés en $I$ conduit à l'expression suivante :

$Q_{S} S /\left(d_{m}^{5 / 2}(\delta-1)^{3 / 2} \delta\right)=$

$$
Y=13,7 \phi(\beta+2)[\alpha \phi-0,047]^{3 / 2}
$$


Nota: Les coefficients numériques 21 et 13,7 qui interviennent dans les expressions de $\mathrm{K}, \mathrm{X}$ et $\mathrm{Y}$ ont la dimension $L^{1 / 2} T^{-1}$. Les valeurs de ces coefficients peuvent donc être calculées dans un autre système d'unités n'utilisant pas le mètre comme unité de longueur $(L)$ ou la seconde comme unité de temps (T).

\section{Représentation graphique et interpréta- tion}

La figure 1 traduit les expressions (1) et (2) dans les axes $X, \phi$, avec comme paramètres $\beta$ et $Y$. En première approximation, dans l'expression (2), $\alpha$ est supposé égal à 1 .

La figure 2 traduit l'hypothèse $Q_{S}$ maximum par une relation entre $X$ et $Y$ tirée de la figure 1 .

\subsection{Comparaison avec des résultats expérimen- taux}

Les caractéristiques principales de la Rivière des Galets sont les suivantes :

$$
\begin{array}{ll}
- \text { pente } S=2 \% ; & -d_{m}=0,21 \mathrm{~m} ; \\
-d_{90}=0,51 \mathrm{~m} ; & - \text { densité du sédiment } \\
& \delta=2,8 .
\end{array}
$$

Les essais ont été réalisés pour des débits $Q_{L}$ échelonnés de 300 à $3000 \mathrm{~m}^{3} / \mathrm{s}$.

Les valeurs du débit solide trouvées sur le modèle sont comparées dans le tableau ci-après avec les valeurs calculées tirées de la figure $2(\alpha=1)$. A titre indicatif, le calcul a été réalisé pour 2 valeurs de la pente : $S=2 \%$ et $S=1,8 \%$.

\begin{tabular}{|r|c|c|c|r|rr|r|}
\hline \multicolumn{2}{|c|}{ Modèle } & \multicolumn{6}{|c|}{ Calcul } \\
\hline \multirow{2}{*}{$Q_{L} \mathrm{~m}^{3} / \mathrm{s}$} & \multirow{2}{*}{$Q_{S} \mathrm{~m}^{3} / \mathrm{s}$} & \multicolumn{3}{|c|}{$S=2 \%$} & \multicolumn{3}{c|}{$S=1,8 \%$} \\
\cline { 3 - 8 } & & \multicolumn{1}{|c|}{$Y$} & $Q_{S} \mathrm{~m}^{3} \mathrm{~s}$ & \multicolumn{1}{c|}{$X$} & \multicolumn{1}{c|}{$Q_{S} \mathrm{~m}^{3} / \mathrm{s}$} \\
\hline 333 & $\boldsymbol{\varepsilon}$ & 0,86 & 0,27 & $\mathbf{1 , 8 4}$ & 0,69 & 0,2 & 1,5 \\
500 & $\mathbf{1 , 2}$ & 1,29 & 0,46 & $\mathbf{3 , 1 4}$ & 1,04 & 0,35 & 2,6 \\
1000 & $\mathbf{4 , 6}$ & 2,59 & 1,1 & $\mathbf{7 , 5 1}$ & 2,07 & 0,85 & 6,4 \\
2000 & $\mathbf{1 1 , 7}$ & $\mathbf{5 , 1 8}$ & 2,5 & $\mathbf{1 7 , 0 8}$ & 4,15 & 1,9 & 14,4 \\
3000 & $\mathbf{1 8 , 6}$ & $\mathbf{7 , 7 7}$ & 3,9 & $\mathbf{2 6 , 6 4}$ & 6,22 & 3 & 22,7 \\
\hline
\end{tabular}

On constate, pour $S=2 \%$, des valeurs calculées de $Q_{S}$ supérieures de près de $50 \%$ aux valeurs expérimentales même pour des valeurs fortes de $Q_{L}$; le seuil de début de charriage de l'ordre de $330 \mathrm{~m}^{3} / \mathrm{s}$ sur le modèle est très supérieur à la valeur calculée.

Pour $S=1,8 \%$, la comparaison expérience-calcul est plus satisfaisante mais donne encore des valeurs calculées trop fortes; cette remarque est cependant intéressante car elle montre le poids de la pente $S$ dans les calculs et de ce fait du coefficient minorateur $\alpha$ dans l'expression $B$ du débit solide.

\subsection{Ajustement de l'expression (2)}

Le coefficient minorateur $\alpha$, pris égal à 1 en première approximation, est d'après Meyer-Peter égal à :

$$
\alpha=\left(Q / Q_{L}\right)\left(k_{s} / K\right)^{3 / 2}
$$

avec : $-Q$ débit liquide qui participe au charriage

$-Q_{L}$ débit liquide total

$-k_{s}$ rugosité du lit, dunes comprises

$-K$ rugosité du sédiment

En gardant l'hypothèse du fond plat $\left(k_{s}=K\right), \alpha$ serait donc essentiellement égal à $\alpha=Q / Q_{L}$.

Nota: Pour des faibles débits, l'écoulement est généralement concentré dans un chenal unique; pour des forts débits l'écoulement peut intéresser plusieurs chenaux dont certains, peu profonds, ne participent pas au charriage. Il est donc logique, a priori, de penser que $\alpha$ est une fonction décroissante de $Q_{L}$, c'est-à-dire de $X$ et de $\phi$ (cf. figure 1).

\section{$*$}

Pour permettre une comparaison satisfaisante entre expérience et calcul pour les forts débits, tout en tenant compte du nota précédent, nous avons été amenés à adopter les valeurs suivantes de $\alpha$ :

\begin{tabular}{c|c|c|c|c|c|c|c|c}
$\phi=$ & 0,047 & 0,05 & 0,06 & 0,08 & 0,1 & 0,2 & 0,3 & 0,5 \\
\hline$\alpha=$ & 1 & 0,987 & 0,954 & 0,913 & 0,888 & 0,838 & 0,822 & 0,808
\end{tabular}

La figure 3 (page suiv.) traduit l'expression (1) et l'expression (2) dans laquelle les valeurs de $\alpha(\phi)$ précédentes ont été introduites.

Sur la figure 2 la nouvelle relation entre $X$ et $Y$ qui exprime l'hypothèse de $Q_{S}$ maximum a été tracée à partir de la figure 3; elle est repérée par $\alpha<1$.

\section{3. $2^{e}$ comparaison avec des résultats expéri- mentaux}

Rivière des Galets: La nouvelle relation entre $X$ et $Y(\alpha<1)$ permet d'établir le tableau suivant comparant les valeurs expérimentales et les valeurs calculées :

\begin{tabular}{|c|c|l|l|c|}
\hline \multicolumn{2}{|c|}{ Modèle } & \multicolumn{3}{c|}{ Calcul $(S=2 \%)$} \\
\hline$Q_{L} \mathrm{~m}^{3} / \mathrm{s}$ & $Q_{S} \mathrm{~m}^{3} / \mathrm{s}$ & \multicolumn{1}{|c|}{$\mathrm{X}$} & \multicolumn{1}{|c|}{$\mathrm{Y}$} & $\mathrm{Qs} \mathrm{m}^{3} / \mathrm{s}$ \\
\hline 20 & 0 & 0,0533 & 0 & 0 \\
333 & $\varepsilon$ & 0,86 & 0,185 & 1,2 \\
500 & $\mathbf{1 , 2}$ & 1,29 & 0,315 & $\mathbf{2 , 1}$ \\
1000 & $\mathbf{4 , 6}$ & 2,59 & 0,72 & $\mathbf{4 , 9}$ \\
2000 & $\mathbf{1 1 , 7}$ & 5,18 & 1,65 & $\mathbf{1 1 , 4}$ \\
3000 & $\mathbf{1 8 , 5}$ & 7,77 & 2,65 & $\mathbf{1 8 , 2}$ \\
\hline
\end{tabular}

La comparaison est satisfaisante pour les valeurs de $Q_{L}>500 \mathrm{~m}^{3} / \mathrm{s}$.

Pour $Q_{L}<500 \mathrm{~m}^{3} / \mathrm{s}$ les valeurs calculées sont supérieures aux valeurs expérimentales; deux raisons au moins à celà : 


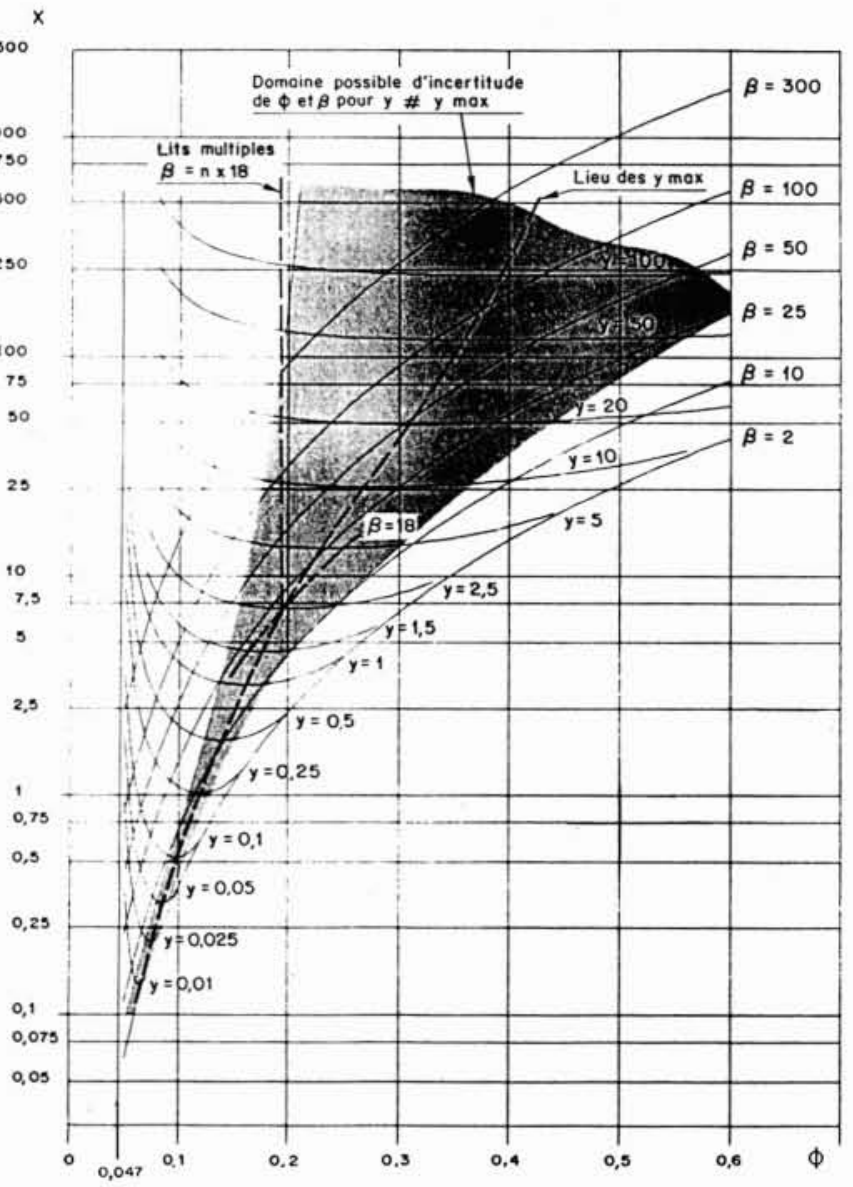

3. $\alpha<1$.

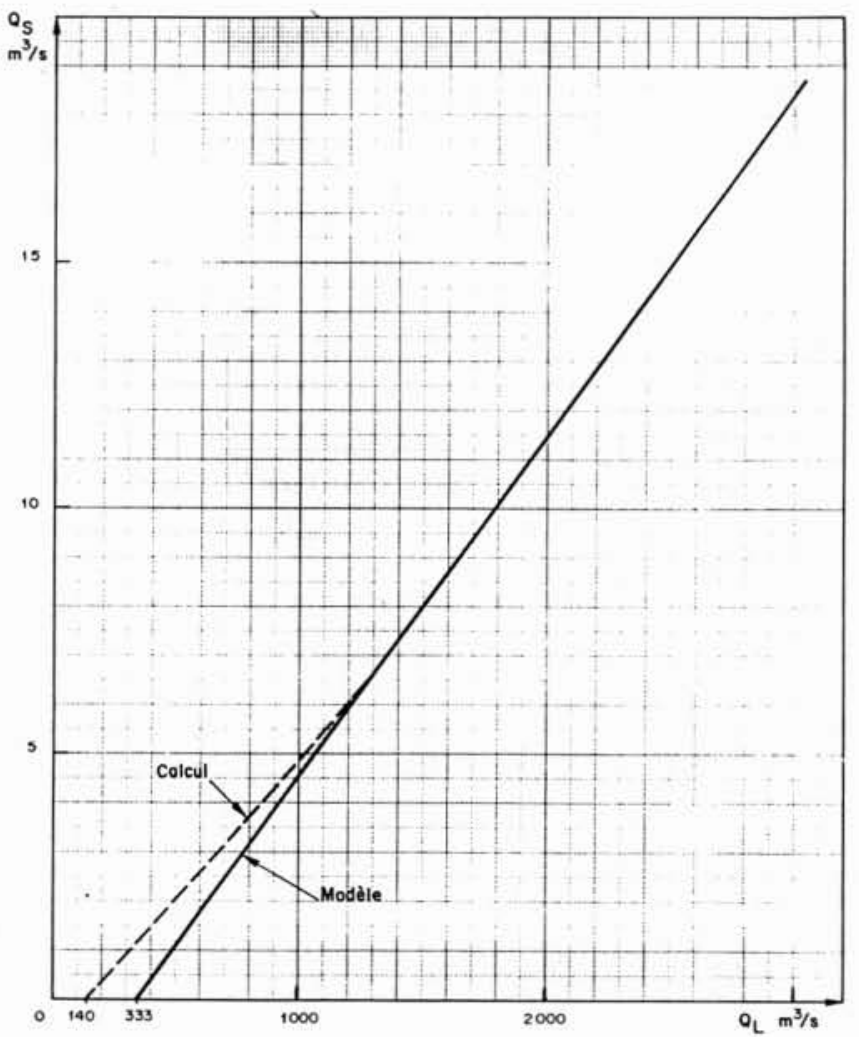

4. Rivière des galets. Relation: débit liquide $Q_{L}-$ débit solide $Q_{S}$.
En premier lieu, pour éviter la formation de rides parasites sur le modèle, la partie la plus fine des sédiments a été éliminée; mais la valeur de $d_{m}$ étant respectée on pouvait supposer que le seuil du début de charriage total serait semblable à celui du prototype. Compte-tenu de l'échelle du modèle, il est cependant possible que pour les très faibles débits une turbulence insuffisante autour des grains puisse ne pas respecter la similitude de tout début d'entraînement.

En second lieu, il est connu que la relation de Meyer-Peter traduit correctement en général le transport solide pour $\phi>0,047$, mais qu'elle est assez imprécise pour $\phi \# 0,047$, c'est-à-dire pour le tout début de charriage total. Pour trancher l'indétermination, nous avons fait l'hypothèse que dès l'instant où le $d_{90}$ de la courbe granulométrique était entraîné, l'ensemble des sédiments était en mouvement et donc que le charriage était total.

Partant d'une relation du type Isbah donnant la vitesse minimale $V$ pour entraîner un bloc de diamètre équivalent $D_{e}$ :

$$
\underline{V=1,2 \sqrt{2 g(\delta-1) D_{e}}}
$$

avec $\delta=2,8$ et $D_{e}=d_{90}=0,51 \mathrm{~m}$ on en déduit $\boldsymbol{V}=\mathbf{5 , 1}$ $\mathbf{m} / \mathbf{s}$.

Sachant par ailleurs (cf. figures 1 ou 3) que le tout début d'entraînement se produit pour une valeur de
$\beta=W / D$ proche de 2 , l'application de la relation de Manning Strickler :

$$
V=K R_{H}^{2 / 3} S^{1 / 2}
$$

avec $K$ exprimé par la relation donnée en $3.1, S=2 \%$, et $R_{H}=W D /(W+2 D)=D / 2$ conduit à :

$$
\begin{aligned}
& R_{H}=1,85 \mathrm{~m} \\
& D=3,7 \mathrm{~m} \\
& W=7,4 \mathrm{~m} .
\end{aligned}
$$

Il y correspond une section $A=W D=27,4 \mathrm{~m}^{2}$ et un débit $Q_{L}=A V=140 \mathrm{~m}^{3} / \mathrm{s}$.

$Q_{L}=140 \mathrm{~m}^{3} / \mathrm{s}$ serait donc le débit correspondant au début de charriage total; cette valeur comprise entre 20 $\mathrm{m}^{3} / \mathrm{s}$ du calcul, certainement trop faible, et $333 \mathrm{~m}^{3} / \mathrm{s}$ de l'expérience, probablement trop forte, paraît donc vraisemblable.

La relation $Q_{L}, Q_{S}$ proposée pour la Rivière des Galets est tracée sur la figure 4 ; elle est comparée à la relation expérimentale.

\section{Autres rivières}

Trois autres rivières ont fait l'objet d'études sur modèle et ont permis d'obtenir quelques valeurs du débit solide; il s'agit de la Rivière des Pluies, la Rivière Saint Etienne et la Rivière des "Patates à Durand". 
Les caractéristiques de ces rivières, les valeurs expérimentales du débit solide et les valeurs calculées à partir de la Figure $2(\alpha<1)$ sont données dans le tableau suivant :

Modèles :

Rivières des Pluies :
Calcul

\begin{tabular}{|c|c|c|c|}
\hline terce a & $X$ & $Y$ & $Q_{s} \mathrm{~m}^{3} / \mathrm{s}$ \\
\hline $\begin{array}{l}S=2,5 \% \quad d_{m}=0,2 \mathrm{~m} \quad \delta=2,8 \\
Q_{L}=1000 \mathrm{~m}^{3} / \mathrm{s} \quad Q_{S}=\mathbf{6 , 5} \mathrm{m}^{3} / \mathrm{s}\end{array}$ & 4,71 & 1,45 & 7 \\
\hline Rivière Saint Etienne & $X$ & $Y$ & $Q_{s} \mathrm{~m}^{3} / \mathrm{s}$ \\
\hline $\begin{array}{l}S=1,8 \% \quad d_{m}=0,1 \mathrm{~m} \quad \delta=2,8 \\
Q_{L}=6000 \mathrm{~m}^{3} / \mathrm{s} \quad Q_{S}=45 \mathrm{~m}^{3} / \mathrm{s}\end{array}$ & 79,5 & 33 & 39 \\
\hline Rivière des "Patates à Durand" & $X$ & $Y$ & $Q_{s} \mathrm{~m}^{3} / \mathrm{s}$ \\
\hline $\begin{array}{l}S=4,8 \% \quad d_{m}=0,3 \mathrm{~m} \quad \delta=2,8 \\
Q_{L}=650 \mathrm{~m}^{3} / \mathrm{s} \quad Q_{S}=6,2 \mathrm{~m}^{3} / \mathrm{s}\end{array}$ & 4,44 & 1,35 & 9,3 \\
\hline
\end{tabular}

Les résultats calculés sont, de 8 à $18 \%$ près, équivalents aux résultats expérimentaux trouvés sur la Rivière des Pluies et la Rivière Saint Etienne; c'est une précision admissible dans ce type d'évaluation.

La divergence par contre est plus importante pour la Rivière des " Patates à Durand ", bien qu'il conviendrait de vérifier les valeurs de $S$ et de $d_{m}$ de cette rivière pour conclure.

On retiendra que la figure $2(\alpha<1)$ donne probablement des valeurs trop importantes du débit solide pour $S>5 \%$; l'exemple de la Tinée dans le $\operatorname{Var}^{(*)}, S \# 10 \%$, semble corroborer cette remarque. Les valeurs de $\alpha$ introduites dans l'équation $B$ seraient probablement à minorer par rapport à celles adoptées pour que calcul et réalité soient davantage en accord pour ces très fortes valeurs de la pente. Les pertes d'énergie du fait des fortes ondulations de surface et les problèmes de pavage ne sont probablement pas étrangers à cette divergence.

\section{Justification de l'hypothèse - Fonds plats}

Dans les études précédentes [1], on met en évidence que des dunes sont censées apparaître entre $\phi=0,062$ et $\phi=2,5$ avec un maximum d'amplitude vers $\phi=0,4$ et ce, quel que soit le rapport entre la dimension du sédiment et la profondeur d'eau $=d / D$.

En fait, les études conduites sur les dunes [3] montrent que la hauteur relative des dunes $\Delta / D$ (rapport entre hauteur de la dune et profondeur d'eau), passe par un maximum de l'ordre de 0,36 .

( ${ }^{\star}$ ) La Tinée a fait l'objet d'une étude sur modèle au $1 / 12,5$ [2]. Le "pavage " observé est probablement responsable en grande partie de la divergence entre calcul et réalité.

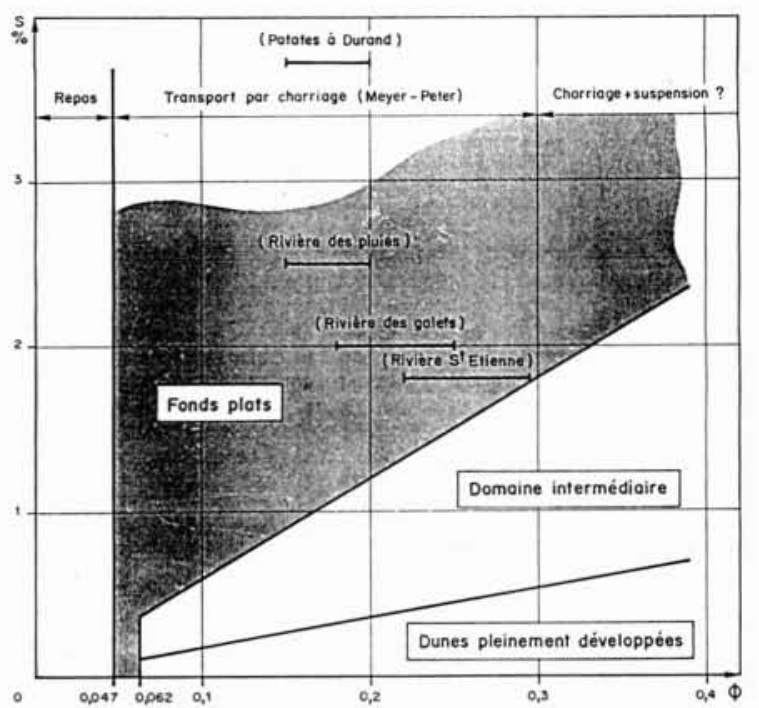

5. Domaines caractéristiques du transport solide.

Il est bien certain que si la dimension maximale du sédiment est de l'ordre de grandeur de $0,36 \mathrm{D}$, aucune dune ne pourra se développer.

Soit $d_{100}$ la dimension maximale du sédiment:

$d_{100}=0,36 D$ représente la condition certaine de non apparition des dunes.

Dans la plupart des cours d'eau naturels, $d_{100}$ est grosso modo égal à 3 à 4 fois $d_{m}$; la relation précédente peut donc s'écrire

$$
d_{m} \# 0,1 D \text { soit } D / d_{m} \# 10
$$

Pour des valeurs de $D / d_{m} \# 30$ la hauteur des dunes, si elles existent, peuvent encore être considérées comme négligeables; pratiquement la condition de non apparition des dunes peut donc s'exprimer par

$$
D / d_{m}<30
$$

Cette relation s'exprime en fonction de $\phi$ en assimilant le rayon hydraulique $R_{H}$ à la profondeur $D$.

soit avec $\delta=2,8$

$$
\phi=D \cdot S /(\delta-1) d_{m}
$$

$$
S>0,06 \phi
$$

On peut alors dresser le tableau suivant donnant la valeur minimale de $S$ pour que les fonds restent plats :

\begin{tabular}{c|c|c|c|c|c|c|}
$\phi=$ & $\begin{array}{c}0,047 \\
\text { à } 0,062\end{array}$ & 0,1 & 0,15 & 0,2 & 0,25 & 0,3 \\
\hline$S$ mini $=$ & qqe & $0,6 \%$ & $0,9 \%$ & $1,2 \%$ & $1,5 \%$ & $1,8 \%$
\end{tabular}

On peut vérifier que dans tous les cas expérimentaux relatés: rivière des Galets, rivière des Pluies, rivière Saint Etienne, rivière des "Patates à Durand", le transport solide se fait sur fonds plats et que la pente $S$ de ces rivières et le domaine de variation de $\phi$ correspondant sont effectivement bien dans la gamme des valeurs données dans le tableau précédent (cf. figure 5 ). 
Nota 1 La figure 2, $(\alpha<1)$, valable uniquement pour des fonds plats, ne peut s'utiliser que pour des pentes $S$ assez importantes: au-dessus de valeurs variant de $0,6 \%$ à plus de $2 \%$ selon $\phi$ Cependant, pour des valeurs de $S$ supérieures à $5 \%$, la figure 2 donnera des valeurs par excès du transport solide.

Nota 2 Pour des pentes inférieures aux valeurs indiquées dans le tableau précédent, des dunes se développeront progressivement; on peut estimer que le développement maximal des dunes peut être atteint lorsque le rapport $D / d_{m}>100$ [d'après Yalin - Réf. 3].

D'où le tableau suivant donnant la valeur maximale de $S$ pour que les dunes soient pleinement développées :

\begin{tabular}{c|c|c|c|c|c|c|}
$\phi=$ & $\begin{array}{c}0,047 \\
\text { à } 0,062\end{array}$ & 0,1 & 0,15 & 0,2 & 0,25 & 0,3 \\
\hline$S \max .=$ & qqe & $0,18 \%$ & $0,27 \%$ & $0,36 \%$ & $0,45 \%$ & $0,54 \%$
\end{tabular}

Dans ce cas le coefficient minorateur $\alpha$ dans la formule $B$ est égale à

$$
\alpha=Q_{L} / Q \cdot\left(K_{s} / K\right)^{3 / 2}
$$

$$
\text { avec } \quad K_{s} / K=\left[\left(0,06+0,41 \phi^{2}\right) / \phi\right]^{5 / 8}[\text { Réf. 1] }
$$

Nota 3 On notera la difficulté du calcul du débit solide $Q_{S}$ pour des rivières dont la pente est intermédiaire entre les valeurs données par les 2 tableaux précédents, c'est-à-dire dont la rugosité des fonds est intermédiaire entre celle correspondant aux fonds plats et celle correspondant aux dunes pleinement développées. Aucune étude expérimentale n'a été entreprise dans ce domaine transitoire; l'indétermination ne peut donc être levée, sinon par des interpolations hardies.

\section{$*$}

La figure 5 représente dans les axes $S, \phi$ les domaines fonds plats, dunes et domaine intermédiaire.

\section{Quelques considérations}

\subsection{Sur la morphologie générale}

Sur la figure 3 , on a tracé les limites du domaine à l'intérieur duquel la condition $Q_{S}$ maximum (ou $Y$ maximum) peut pratiquement, à moins de $10 \%$ près, être considérée comme satisfaite. On constate que ce domaine s'élargit considérablement vers les fortes valeurs de $X$, c'est-à-dire au fur et à mesure que le débit liquide augmente.

Pour les fortes valeurs du débit, le lit peut donc être très large ( $\phi$ faible et $\beta$ grand) ou beaucoup plus étroit : les deux morphologies peuvent donc cohabiter et c'est effectivement un fait d'observation courante en période de crue pour des torrents du type "Rivière des Galets " : à un lit très large sur un certain bief peut succéder un chenal relativement étroit dans un autre bief, l'une et l'autre des configurations peuvent transporter la même valeur du débit liquide et du débit solide tout en satisfaisant pratiquement la condition de transport solide maximum.

Par contre, pour les faibles valeur de $X$, c'est-à-dire du débit, les limites du domaine se rétrécissent du fait des minima très prononcés que présentent les courbes
$Y=$ cste; elles tendent vers la courbe $\beta=2$ lorsque $\phi$ tend vers 0,047 , c'est-à-dire pour le tout début de charriage : en étiage, le lit étroit et relativement profond concentre la quasi totalité du débit; c'est là aussi un fait d'observation courant.

\subsection{Sur la réalisation d'endiguements}

Tout endiguement a évidemment comme fonction première de concentrer le débit, c'est-à-dire qu'il entraîne généralement une augmentation du coefficient $\alpha$ qui tend vers 1 (cf. chapitre IV.2). Le lit endigué a de ce fait une capacité de charriage plus importante que celle du lit laissé à l'état naturel, à l'amont des ouvrages en particulier.

La pente à l'intérieur de l'endiguement peut donc être plus faible que celle du lit naturel pour assurer le transit du débit solide, qui lui vient d'amont; il en résulte un risque d'érosion régressive non négligeable.

A titre indicatif, on a représenté sur la figure 2 les courbes iso débits solides calculées entre la courbe $Y=f(X)$ pour $\alpha<1$ correspondant au lit large naturel et la courbe $Y=f(X)$ pour $\alpha=1$ qui correspondrait théoriquement au lit endigué. Il est ainsi possible d'évaluer la diminution des paramètres $X$ et $\mathbf{Y}$, c'est-à-dire en fait de la pente $S$ qu'entraîne un endiguement trop étroit. On vérifiera qu'à débit solide constant le rapport entre la pente $S_{1}$ du lit endigué $(\alpha=1)$ et la pente $S_{0}$ du lit naturel $(\alpha<1)$ est égal à $Y_{1} / Y_{0}$ et est de l'ordre de 0,8 .

L'endiguement à réaliser doit donc constituer un compromis entre l'intérêt de récupérer les terrains inondables et le risque de voir apparaitre une érosion régressive importante dommageable pour les ouvrages implantés en amont.

Compte-tenu des remarques précédentes, un premier avant projet d'endiguement peut être réalisé en prenant en compte la limite inférieure du domaine représenté sur la figure 3 et qui correspond aux plus grandes valeurs de $\beta$, c'est-à-dire aux plus grandes largeurs du lit naturel répondant aux critères de stabilité et de capacité de transit des débits liquides et solides.

A titre d'exemple, sur la Rivière des Galets, au débit maximum envisagé de $3000 \mathrm{~m}^{3} / \mathrm{s}$ correspond la valeur $X \# 8$ (cf. $\S 4.3$ ).

A cette valeur de $X$, les valeurs de $\phi$ et $\beta$, données par la figure 3 , sont respectivement :

$$
\begin{aligned}
& \phi=0,155 ; \\
& \beta=50 .
\end{aligned}
$$

En se reportant aux définitions de $\phi$ et de $\beta$ et compte-tenu des données spécifiques de la Rivière des Galets : $S=2 \%, \delta=2,8, d_{m}=0,21 \mathrm{~m}$, on trouve :

largeur $W \# 150 \mathrm{~m}$;

profondeur $D \# 3 \mathrm{~m}$;

on en déduit $K=24,4$.

Ces caractéristiques peuvent être envisagées pour réaliser l'endiguement de la Rivière des Galets; elles ont fait l'objet de tests sur modèle physique qui en ont démontré le bien fondé sur le plan technique.

Cependant, l'étude approfondie sur modèle d'autres solutions encadrant cette solution possible, ont conduit 
à adopter un endiguement plus large, de l'ordre de 200 mètres.

Les vitesses moyennes, plus faibles dans un tel endiguement, diminuent en effet l'importance des protec- tions à mettre en place pour faire face aux affouillements en pied de digue et aux érosions de talus, et en conséquence rendent le projet économiquement plus intéres. sant.

\section{Conclusion}

La relation $Y=f(X)$, avec $\alpha<1$, donnée sur la figure 2 permettra dans la plupart des cas de rivières à fortes pente, à l'intérieur du domaine "transport solide sur fond plat " défini sur la figure 5 , d'évaluer le débit solide de ce type de rivières pour un débit liquide donné.

L'association d'une étude hydrologique et de la relation débit solide-débit liquide permettra d'évaluer les volumes d'apport solide annuels correspondant à telle ou telle probabilité d'année hydrologique et, en conséquence, pourra servir de base à une politique d'extraction de graviers.
Les caractéristiques d'un endiguement respectant au mieux les paramètres morphologiques de la rivière naturelle en crue : largeur, profondeur, pente, pourront être évaluées en première approximation à partir de la figure 3. Pour limiter tout risque de basculement de pente et donc d'érosion régressive, la limite inférieure du domaine donnée sur cette figure sera choisie de préfé. rence.

Le choix de l'endiguement définitif, qui résulte d'un compromis technico-économiques entre différentes solutions encadrant la solution théorique, sera toujours avantageusement fait à partir d'une étude sur modèle physique, qui peut seul prendre en compte les phénomènes locaux alors que tout modèle théorique ne peut décrire que des phénomènes morphologiques moyens.

\section{Références}

[0] Etudes sur modèle réduit physique de l'endiguement de la Rivière des Galets - Duvoisin - Rapports SOGREAH de 1985 à 1987 .

[1] Réflexions sur les lois de la morphologie fluviale - Ramette - Rapport LNH E40/84.03 - 1984.

[2] La Tinée - Variation de la granulométrie de charriage dans une section de rivière - Nizery-Braudeau - Proceedings MIHC - 1953 .

[3] Guide d'hydraulique fluviale - Ramette - Rapport LNH E40/81-04 - 1981.

Adresse de l'auteur 